This item was submitted to Loughborough's Research Repository by the author.

Items in Figshare are protected by copyright, with all rights reserved, unless otherwise indicated.

\title{
Robotic-assisted internal fixation of hip fractures: a fluoroscopy-based intraoperative registration technique
}

PLEASE CITE THE PUBLISHED VERSION

PUBLISHER

Professional Engineering Publishing / @ IMECHE

VERSION

VoR (Version of Record)

LICENCE

CC BY-NC-ND 4.0

\section{REPOSITORY RECORD}

Browbank, lan, Kaddour Bouazza-Marouf, and J. Schnabler. 2019. "Robotic-assisted Internal Fixation of Hip Fractures: A Fluoroscopy-based Intraoperative Registration Technique". figshare. https://hdl.handle.net/2134/5144. 
This item was submitted to Loughborough's Institutional Repository (https://dspace.lboro.ac.uk/) by the author and is made available under the following Creative Commons Licence conditions.

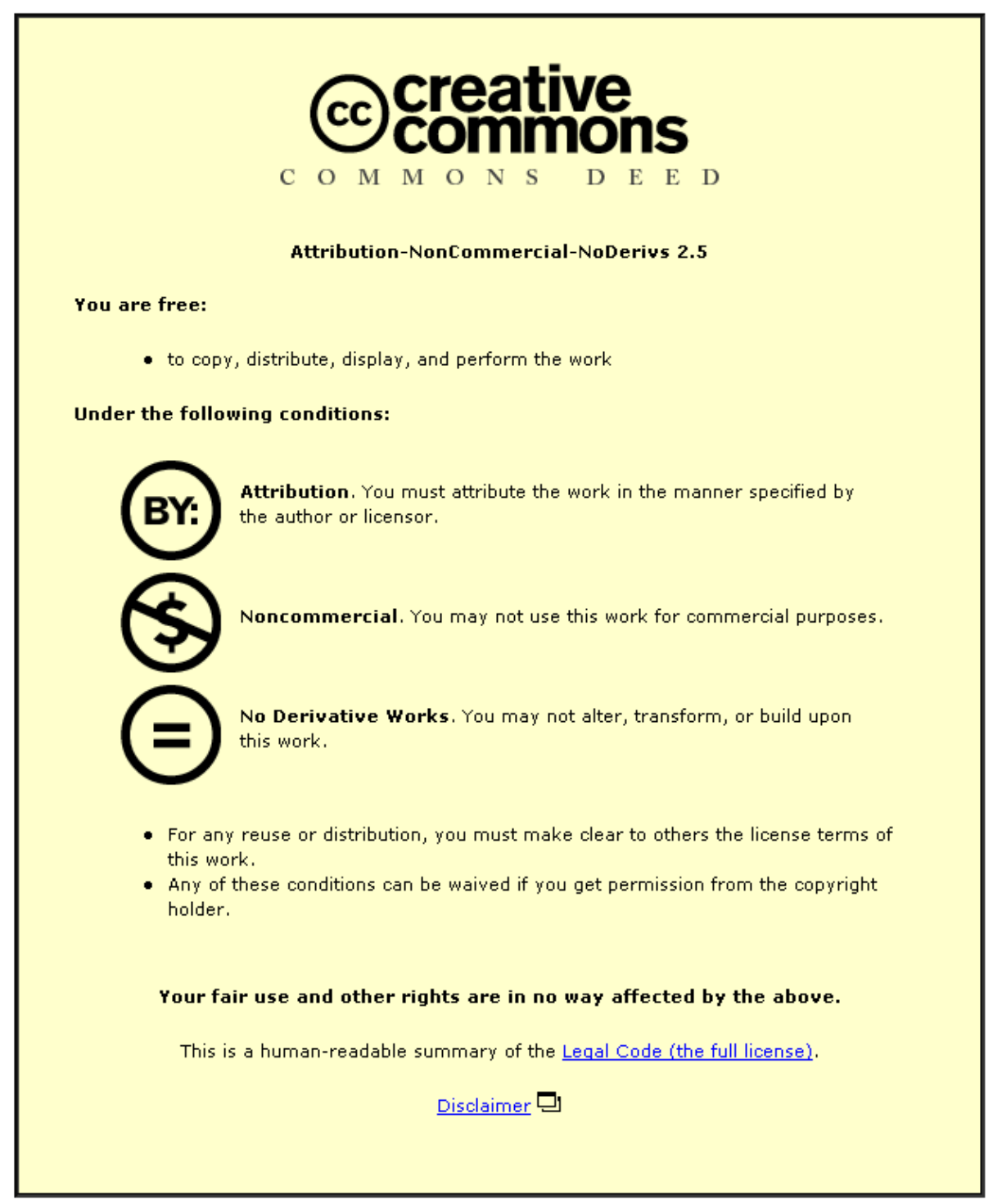

For the full text of this licence, please go to: http://creativecommons.org/licenses/by-nc-nd/2.5/ 


\title{
Robotic-assisted internal fixation of hip fractures: a fluoroscopy-based intraoperative registration technique
}

\author{
I Browbank, K Bouazza-Marouf* and J Schnabler \\ Department of Mechanical Engineering, Loughborough University, Leicestershire UK
}

\begin{abstract}
The internal fixation of proximal femoral (hip) fractures is the most frequently performed orthopaedic surgery procedure. When using a sliding compression hip screw, a commonly used fixation device, accurate positioning of the device within the femoral neck-head is achieved by initially drilling a pilot hole. A cannulated component of the hip screw is then inserted over the guide wire (surgical drill bit), which is used to drill the pilot hole. However, in practice, this fluoroscopically controlled drilling process is severely complicated by a depth perception problem and, as such, a surgeon can require several attempts to achieve a satisfactory guide wire placement. A prototype robotic-assisted orthopaedic surgery system has therefore been developed, with a view to achieving accurate rightfirst-time guide wire insertions. This paper describes the non-invasive digital X-ray photogrammetrybased registration technique which supports the proposed robotic-assisted drilling scenario. Results from preliminary laboratory (in vitro) trials employing this registration technique indicate that the cumulative error associated with the entire X-ray guided robotic system is within acceptable limits for the guide wire insertion process.
\end{abstract}

Keywords: robotic-assisted orthopaedic surgery, intraoperative registration, digital X-ray photogrammetry

\section{INTRODUCTION}

The generic term 'hip fracture' is used to describe any fracture at the proximal (upper) end of the femur. Given that the femur (thigh bone) is the strongest bone in the human body such fractures are rare in adults under the age of 50 years old [1]. However, due to a combination of age-related factors, the most notable of which are the effects of osteoporosis and an increased risk of sustaining a fall, above the age of 50 the incidence of hip fractures increases exponentially and eventually reaches very high rates of fracture among people in their seventies and eighties [2]. In view of the advanced age of a typical patient, the hip fracture constitutes a very serious injury and is associated with high rates of morbidity and mortality.

The orthopaedic treatment of hip fractures involves a three-stage process: reduction, stabilization and bone healing. The term reduction refers to the restoration of

The MS was received on 13 January 1999 and was accepted after revision for publication on 18 March 1999.

* Corresponding author: Department of Mechanical Engineering, Loughborough University, Loughborough, Leicestershire LE11 3TU, $U K$.

H00199 (C) IMechE 2000 the broken bone fragments to their anatomically correct positions, a process which is frequently complicated by rotation of the displaced femoral head relative to the shaft. Having obtained axial and rotational alignment of the broken bone fragments, a suitable stabilization technique is then applied to ensure that reduction is maintained throughout the natural bone healing process. Over a period of months, the continuity of the proximal femur is then restored by the formation and progressive hardening of a callus at the fracture site.

In relation to hip fractures, the preferred fracture stabilization technique is internal fixation (osteosynthesis). Internal fixation procedures involve the surgical insertion of mechanical fixation devices (e.g. screws, pins, plates or nails) into the broken bone fragments. These fixation devices then remain inside the patient for the duration of the bone healing process and are ideally removed following radiographic confirmation of solid union of the fracture site. The type of fixation device(s) used during a hip fracture procedure depends upon the location of the fracture site. The most common site for a hip fracture is in the trochanteric region [3], i.e. near but outside the capsule of the hip joint. The implant design which achieves the best results with these extra- 
capsular hip fractures is the sliding compression hip screw.

As can be seen from Fig. 1, the sliding compression hip screw is a two-piece device consisting of a lag screw and an angled plate. The cannulated lag screw is designed to be inserted into the femoral neck-head with the aid of a guide wire (pilot drill bit). The angled plate is then attached to the lateral cortex of the proximal femur, using a series of screws, with its barrel containing the unthreaded portion of the lag screw. As such, the lag screw is free to slide within the barrel of the angled plate, thus allowing controlled impaction of the fracture site, while at the same time maintaining a constant femoral neck-shaft angle (typically $135^{\circ}$ ).

Although the sliding compression hip screw provides more reliable stabilization than any other osteosynthesis device, post-operative follow-up studies frequently reveal fixation failure rates of the order of 10 per cent. In practice, the majority of these fixation failures tend to occur as a result of the lag screw cutting through the femoral head-neck [4]. By evaluating radiographs taken immediately after surgery, several studies have been able to demonstrate that the probability that such a 'cut-out' occurs is directly related to the initial position of the lag screw within the femoral head [4-8]. In other words, the accuracy of the lag screw insertion process, which in turn is dictated by the accuracy of the guide wire insertion process, effectively dictates the outcome of the surgical procedure.

Unfortunately, as outlined in Section 2, the existing guide wire insertion process is severely complicated by a depth perception problem. In an attempt to improve the accuracy and repeatability of the internal fixation of hip fractures, the robotic-assisted surgery scenario described in Section 3 has therefore been investigated $[9,10]$. In order to implement such a scenario, a registration technique must initially be employed to establish the intraoperative locations, with respect to a common coordinate system, of the patient, the X-ray imaging equipment and the robotic manipulator. A trajectory planning process must then be performed with respect to this common coordinate system. With a view to providing a clinically acceptable solution to these problems, an intraoperative registration technique based upon $\mathrm{X}$-ray photogrammetry principles has been developed. Section 4 therefore provides an in-depth discussion of the acquisition and rapid analysis of digital X-ray 'registration' images.

\section{THE GUIDE WIRE INSERTION PROBLEM}

Assuming that satisfactory reduction of the fracture site can be obtained, the internal fixation of a hip fracture using a sliding compression hip screw will proceed as follows [11]:

(a) surgical access (skin incision and muscle retraction);

(b) guide wire insertion;
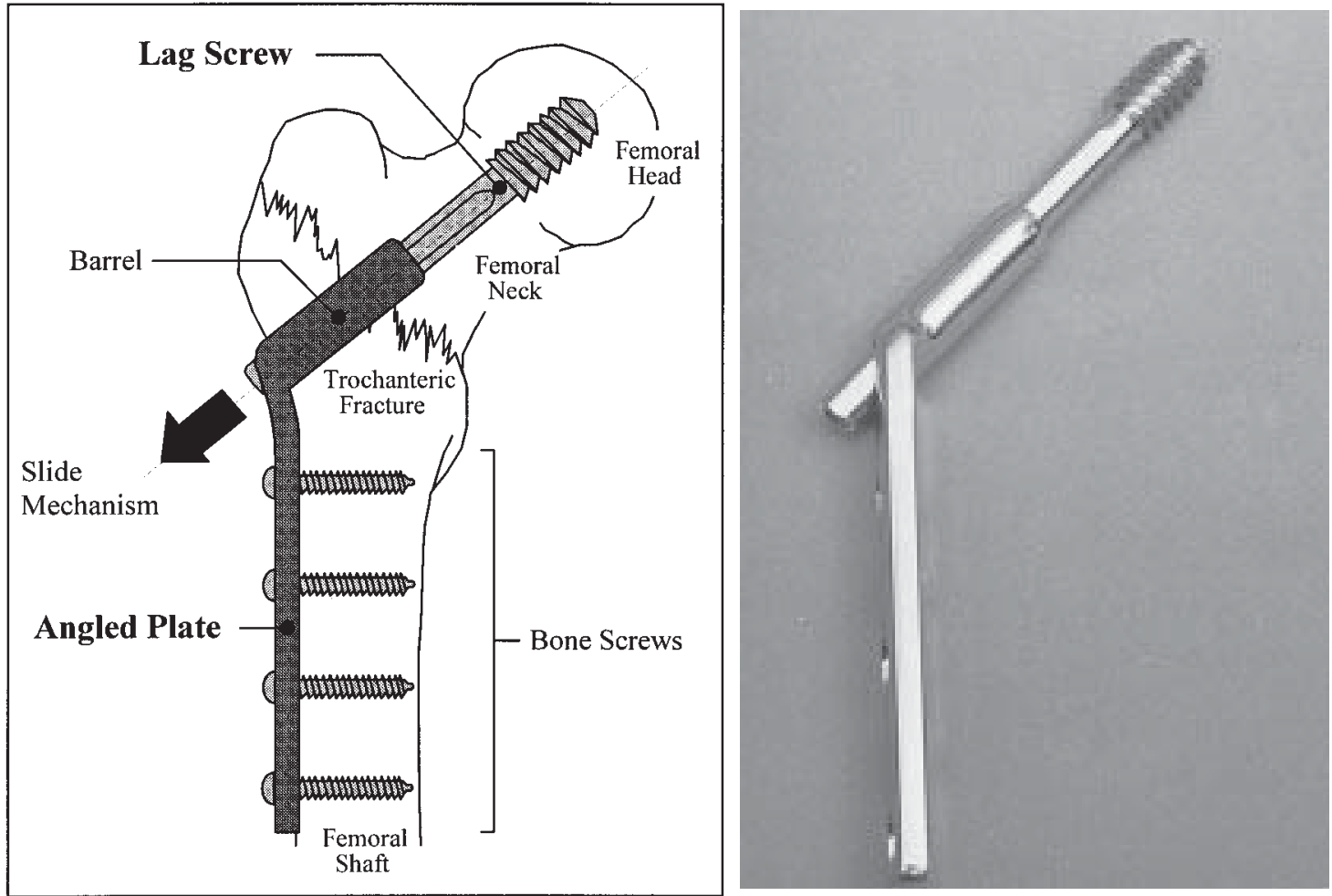

Fig. 1 Internal fixation of a hip fracture using a sliding compression hip screw 
(c) reaming and tapping of the proximal femur (over the guide wire);

(d) insertion of the lag screw (over the guide wire) (the guide wire is then removed);

(e) insertion and attachment (using four screws) of the angled plate.

As previously described, with regard to post-operative results, the guide wire insertion process is the most critical of these invasive stages. A typical guide wire consists of a stainless steel rod 2-3 $\mathrm{mm}$ in diameter and approximately $200 \mathrm{~mm}$ long, with a threaded trocar tip. As a prerequisite to satisfactory lag screw placement, this specialized drill bit must be inserted into the femoral neck-head at a fixed angle to the femoral shaft (usually $135^{\circ}$ ), and to an appropriate depth. As a consequence, having mounted the guide wire in an air-powered surgical drill, it is generally inserted with the aid of a handheld angle (drill) guide which is held in contact with the lateral femoral cortex.

The difficulties currently experienced by orthopaedic surgeons when attempting to insert a guide wire arise due to an unavoidable reliance upon intraoperative $\mathrm{X}$-ray imaging. Once the tip of the guide wire has been drilled into the promixal femur, there is obviously no direct visualization of its trajectory. Intraoperative X-ray imaging (fluoroscopy) therefore provides the only means of monitoring the progress of the guide wire as it is advanced into the femoral neck-head. As a result, the guide wire insertion process is currently performed in the manner shown in Fig. 2.

The patient is positioned on an orthopaedic fracture table in such a way as to allow a mobile C-arm fluor- oscopy unit full access to the fractured hip. This intraoperative X-ray imaging device consists of an X-ray tube housing and an image intensifier housing mounted directly opposite one another on a C-shaped arc. By rotating this arc through $90^{\circ}$, it is possible to obtain both postero-anterior (PA) (back-to-front) and lateral-tomedial (side-to-side) views of the fractured hip.

However, the use of two-dimensional X-ray images to guide a three-dimensional surgical drilling process incurs a depth perception problem. A trial-and-error insertion technique is therefore currently adopted, with the guide wire's progress being monitored at intermediate stages by acquiring both a PA and a lateral-to-medial view. Unfortunately, inexperienced orthopaedic surgeons can encounter great difficulties when trying to perform this highly subjective fluoroscopically guided insertion technique. Given that an individual's hand-eye coordination and visuospatial ability are inherent skills, it is also possible that an individual surgeon's performance may not improve with experience. As a consequence, it is not unusual for several attempts to be required in order to achieve satisfactory positioning of the guide wire.

This failure to perform right-first-time guide wire insertions has many implications. As each subsequent attempt prolongs the duration of the surgical procedure, the patient's intraoperative blood loss and the risk of infection are both increased. The additional anaesthetic received by the patient also has a detrimental effect upon post-operative recovery. However, it is the fact that a less than 'ideal' guide wire placement may eventually have to be accepted after several insertion attempts, leading to an increased probability of fixation failure, which

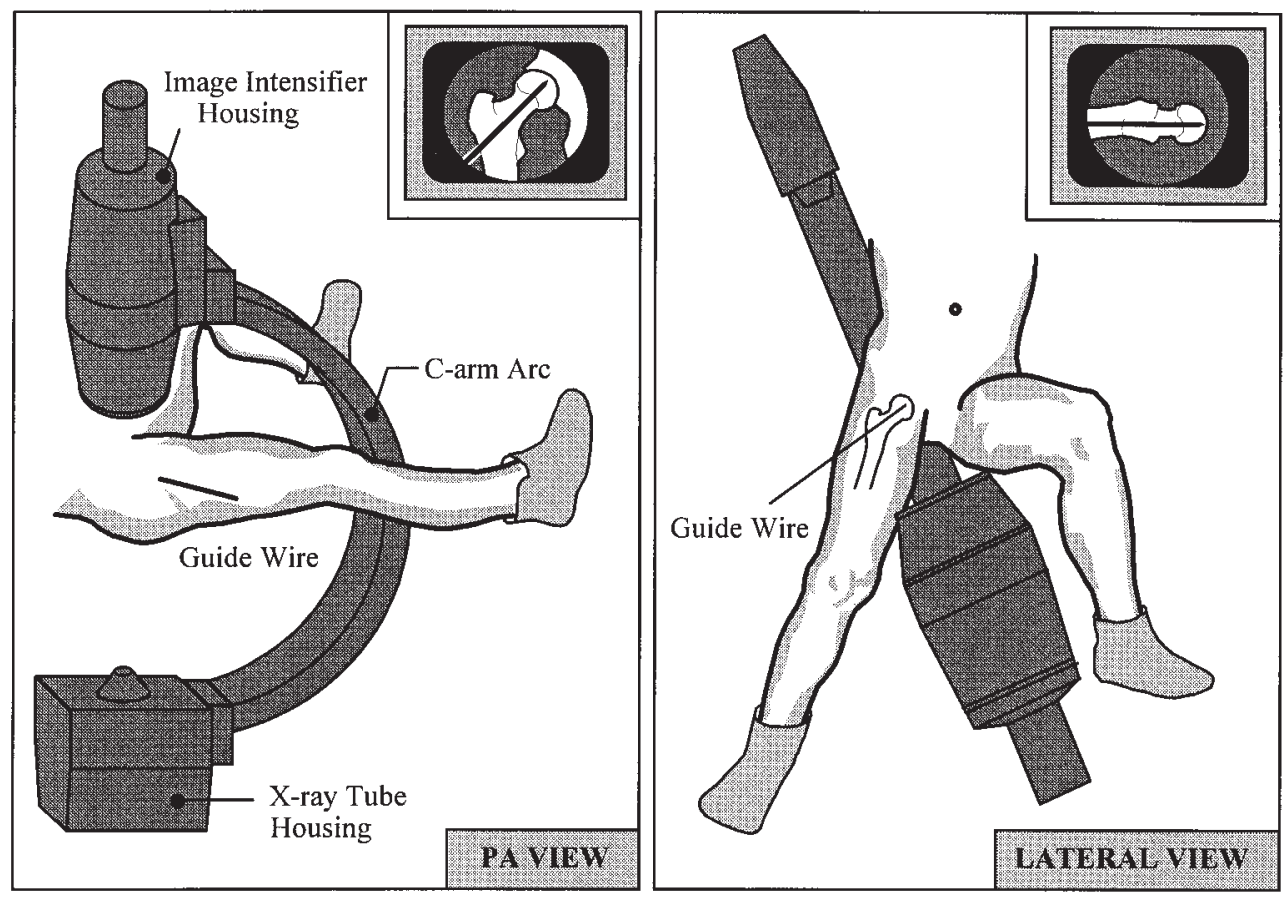

Fig. 2 Fluoroscopic guidance of the guide wire insertion process 
gives the most cause for concern. Therefore, in relation to accuracy and repeatability, the existing guide wire insertion technique leaves considerable scope for improvement.

\section{ROBOTIC-ASSISTED GUIDE WIRE INSERTIONS}

Under the proposed surgical scenario, only the guide wire insertion stage of the hip fracture repair procedure has been targeted for robotic assistance. The robotic manipulator will therefore be introduced following reduction of the hip fracture and will be coarsely aligned with the patient's injured hip. Intraoperative registration and trajectory planning will then be established using the fluoroscopy-based techniques described in Section 4. Computer-controlled fine positioning of the manipulator will then allow alignment of an actuated drilling unit end effector, or a manipulator-mounted drill guide, with this ideal drilling trajectory [10]. Having successfully inserted the guide wire, the manipulator will be removed from the vicinity of the patient, thus allowing the remaining stages of the procedure to be carried out in the usual manner by the orthopaedic surgeon.

Because of the overriding need to maintain patient and surgical staff safety and to comply with surgical sterility criteria, the intraoperative use of a commercially available robot is not advisable. A custom-built robotic manipulator has therefore been manufactured, with a view to performing a laboratory-based investigation of the robotic-assisted guide wire insertion scenario described above [10]. In addition to providing the four degrees of freedom required to align a drill guide with a pre-computed drilling trajectory, the application specific kinematic configuration of this prototype manipulator also incorporates an automatic drilling unit (fifth degree of freedom). A strain gauge force sensor within this drilling unit allows quantification of the drilling process, through the provision of force feedback data. Initial test results indicate that the use of force feedback could play a major role in the safety protocol for an automated guide wire insertion scenario [12].

The prototype robotic system is illustrated in Fig. 3, which shows the integration of the whole system. In addition to the manipulator, the other major system component is a PC-based machine vision unit, which consists of a frame grabber card and procedure specific software modules. By establishing a video link between this frame grabber card and the C-arm unit it is possible to sample the video signal from the image intensifier housing, thus allowing 'digital' X-ray images to be acquired intraoperatively. Having acquired the standard PA and lateral images in a digital format, image enhancement and analysis algorithms can then be applied to extract the appropriate registration data rapidly. A more detailed account of the practicalities of this registration process is provided in the remaining sections of this paper.

\section{INTRAOPERATIVE REGISTRATION}

The proposed robotic-assisted surgery scenario requires a robotic manipulator to position a guide wire (or a drill guide) accurately in relation to the patient's skeletal anatomy (proximal femur). Since direct access to the patient's skeleton is limited to the extent of the surgical incision, the patient's location with respect to the robotic manipulator must be determined indirectly using X-ray images. This calibration or registration process therefore requires the extraction of three-dimensional measurements from two-dimensional X-ray images and, as such, falls within the scope of the X-ray photogrammetry field of research.

X-ray photogrammetry techniques work by introducing artificial calibration features (fiducials) into the standard radiographic views. The acquisition of these calibration images is generally achieved by surrounding the anatomical region of interest with a radiolucent framework, into which a regular array of radiopaque (typically metallic) fiducial markers have been inlaid. In practice, the patient-calibration frame combination will then be imaged using one of the imaging geometries illustrated in Fig. 4. Knowledge of the actual dimensions of the fiducial arrays, coupled with their measured image coordinates, then allows calibration of the photogrammetry system. A triangulation-based reconstruction technique can then be applied to reconstruct the threedimensional locations of target points appearing in the imaged anatomy.

The application of X-ray photogrammetry principles to the guide wire insertion process has led to the adoption of the registration scenario depicted in Fig. 5. Under this scenario, the manipulator is used to hold an anatomically compatible calibration frame in the vicinity of the patient's proximal femur (not shown in Fig. 5). PA and lateral-to-medial images of this patient-frame combination can then be acquired by the C-arm fluoroscopy unit, thus allowing a registration strategy based upon three arbitrarily allocated coordinate systems to be implemented.

The most important of these frames of reference is the MCS, which effectively defines the common (operating room) coordinate system. Accordingly, the ultimate aim of the registration-trajectory planning process is to accurately define drilling trajectories with respect to this MCS. In accordance with most robotic applications, the origin of the MCS has been allocated to a non-moving part of the manipulator's base. Following calibration of the manipulator, the location of the end effector can therefore be calculated in real time, with respect to the MCS, by applying forward kinematics to the manipulator's encoder readings. 


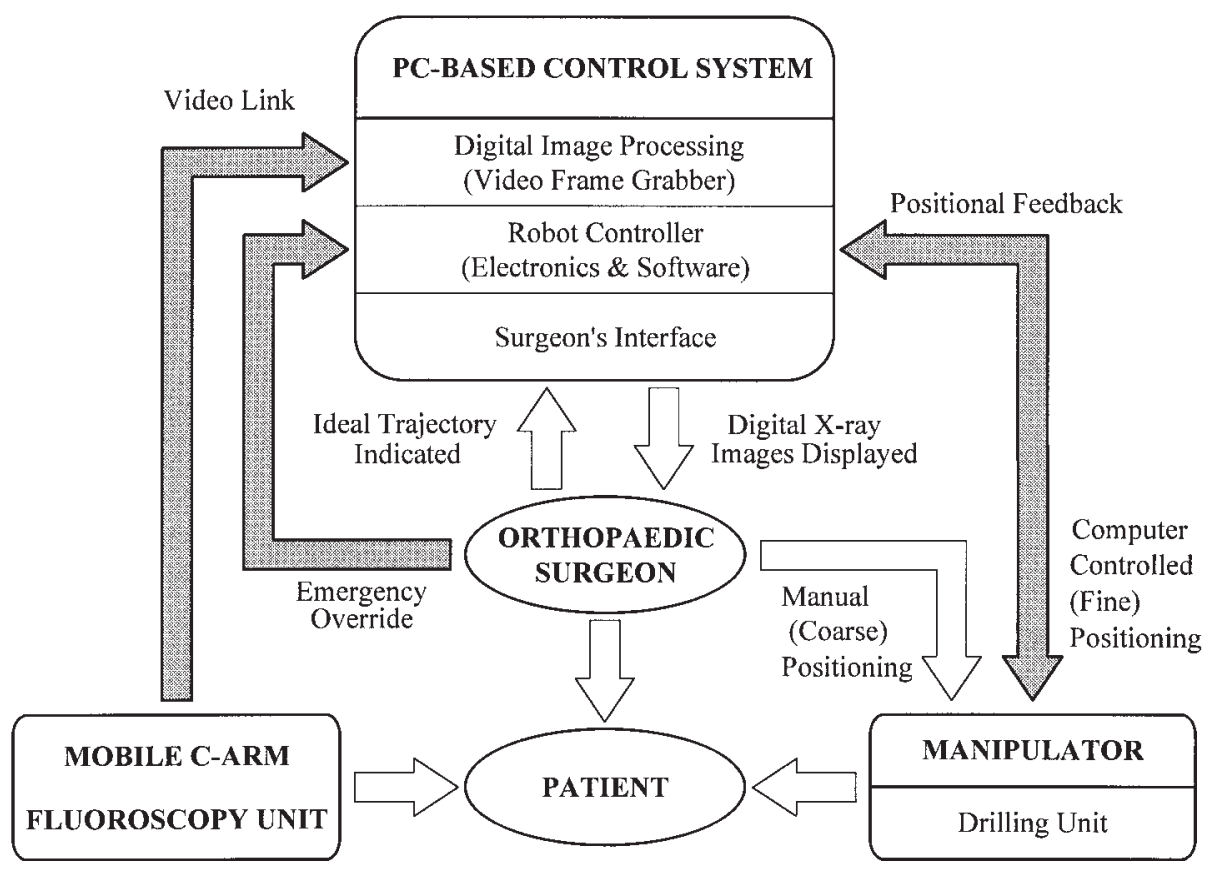

Fig. 3 Block diagram of the prototype robotic system (PC, personal computer)
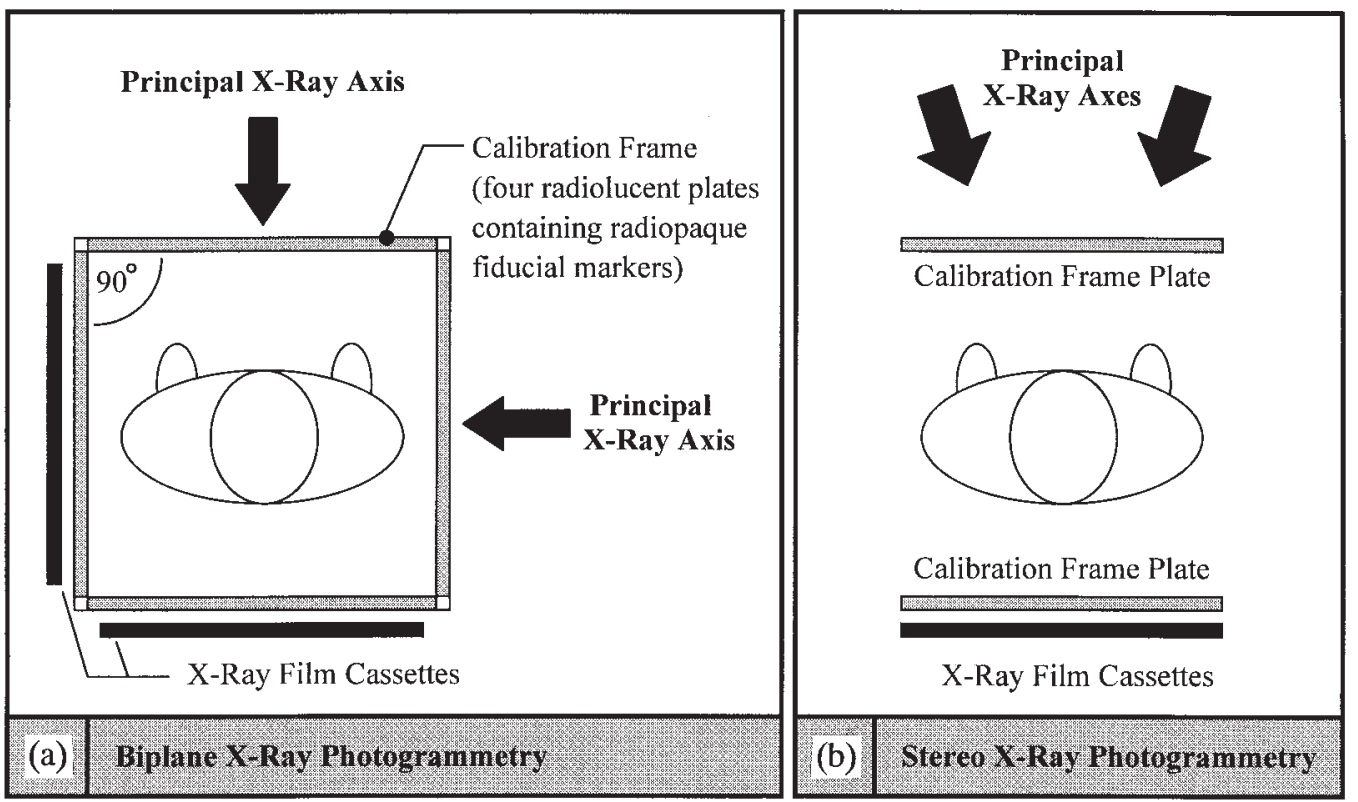

Fig. 4 X-ray photogrammetry imaging geometries

Since the radiolucent calibration frame is rigidly attached to the end effector of the manipulator, a spatial transformation can be established between the CFCS and the MCS. Given that the CFCS is defined by the radiopaque fiducial markers embedded in the calibration frame, and that these fiducial markers are superimposed on the fluoroscopic images, the calibration frame therefore fulfils an intermediary role, allowing an imageworld (or vice versa) transformation to be determined indirectly.

However, in order to implement this registration scen- ario, problems which are not encountered during conventional X-ray photogrammetry applications have had to be addressed. As illustrated in Fig. 4, the vast majority of X-ray photogrammetry applications are radiography (X-ray film) based. Owing to the delay incurred by the film development process, such a technique is obviously unsuitable for intraoperative use. In order to avoid prolonging the surgical procedure, the more technically demanding option of fluoroscopy-based digital X-ray photogrammetry has therefore been investigated. As discussed in Section 4.1, the adoption of this methodology 


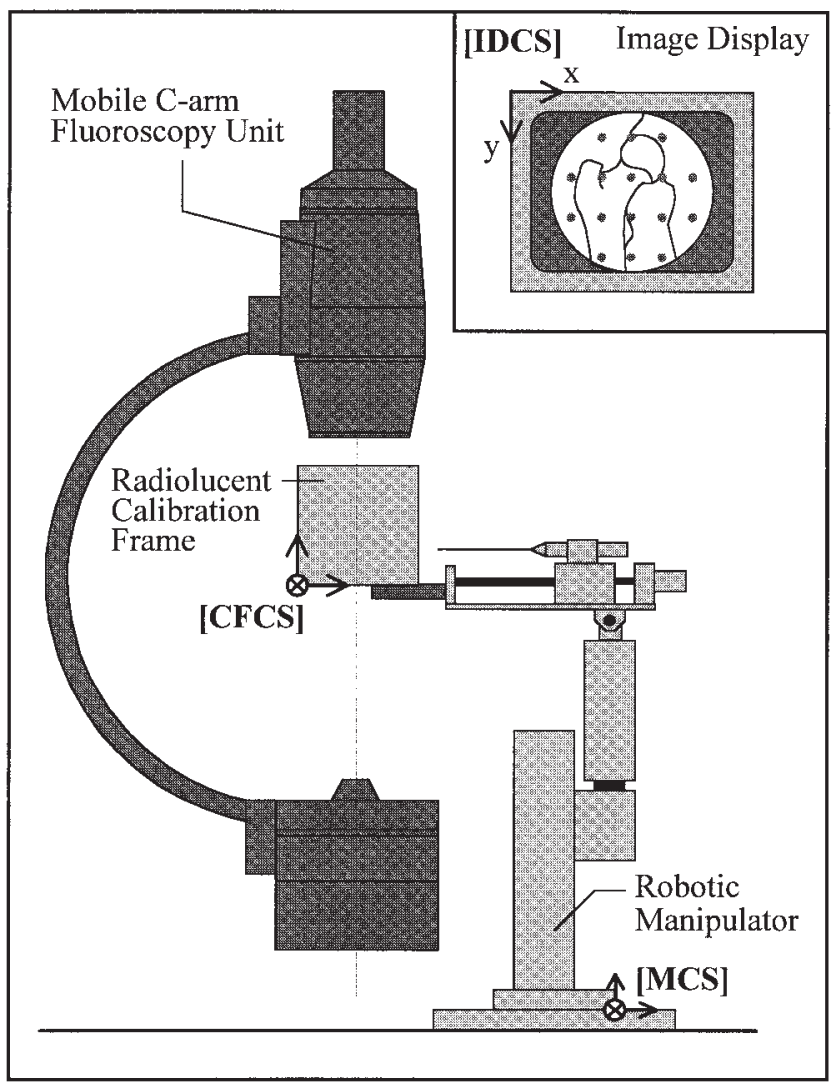

Fig. 5 The fluoroscopy-based intraoperative registration scheme (IDCS, image display coordinate system; CFCS, calibration frame coordinate system; MCS, manipulator coordinate system)

initially raises a number of interesting problems in relation to the provision of a suitable calibration frame.

The use of a frame grabber to digitize the intraoperative fluoroscopic images means that the imaged locations of the fiducial markers are measured in terms of pixels, with respect to an IDCS. The first task of the registration-trajectory planning process is to extract these measurements from the relevant registration images. Semi-automatic image analysis algorithms (outlined in Section 4.2) have therefore been developed to allow rapid intraoperative data extraction. Having extracted this 'raw' image data, an image distortion compensation technique (described in Section 4.4) must then be applied, in order to correct for the highly non-linear imaging properties of the $\mathrm{C}$-arm fluoroscopy unit. The trajectory planning process can then be performed with the aid of the image matching technique described in Section 4.3.

\subsection{Calibration frame design}

The proposed intraoperative use of an X-ray photogrammetry calibration frame, in close proximity to the patient, requires a frame design that can be either iso- lated or sterilized. For simplicity, isolation of the calibration frame is the preferred option. Covering the frame in sterile plastic draping, a technique which is currently employed to isolate the X-ray source and image intensifier housings of the $\mathrm{C}$-arm unit, prevents contamination by isolating the frame from the 'sterile' areas of the operating room. However, if the use of isolation draping proves to be problematic, a preliminary investigation has also shown that an 'autoclave-compatible' calibration frame can be manufactured from materials with appropriate thermal, corrosion resistance and water absorption properties.

In addition to the sterility issue, a $\mathrm{C}$-arm compatibility problem has also had to be addressed. Given that surrounding the lower torso with a four-sided calibration frame is not a viable option, conventional X-ray photogrammetry studies of the hip joint employ the stereo imaging geometry depicted in Fig. 4b [13-15]. Unfortunately, a feasibility study revealed that the imaging geometry of the mobile C-arm fluoroscopy unit is not well suited to stereo X-ray photogrammetry of the hip. The bulky calibration frame which is required to support the stereo configuration also leads to compatibility problems in relation to both the robotic manipulator and the operating table.

In order to provide an 'operating-room-compatible' method of quantifying the fluoroscopic examination process, an alternative to the 'classical' stereo X-ray photogrammetry technique has therefore had to be developed. As can be appreciated from Fig. 6, the calibration frame which supports this new examination technique employs an inverted L-shaped structure to introduce fiducial markers into the standard (PA and lateral-to-medial) fluoroscopic images. As a consequence, the term 'gamma frame' or ' $\Gamma$ frame' has been adopted to describe this configuration.

Positioning this anatomically compatible $\Gamma$ frame around the patient, in the manner shown in Fig. 7 introduces a pair of calibration plates above the hip, and a second pair of calibration plates to the lateral side of the hip. Given that the patient's proximal femur is not located between these calibration plates, it does not lie within the true calibrated volume. As a consequence, the subsequent photogrammetric reconstruction process involves a series of extrapolation-based calculations and, as such, deviates from established photogrammetric principles.

Although this extrapolation-based technique is not as accurate as the classical (interpolation-based) X-ray photogrammetry approach, test results (discussed in Section 5) indicate that the resulting loss of precision is 'acceptable' in relation to the accuracy requirements of the guide wire insertion process. The $\Gamma$ frame therefore represents a 'compromise' solution which provides reconstruction errors of the order of $1 \mathrm{~mm}$ (or better), while at the same time offering significant advantages in terms of intraoperative ease of use (i.e. quicker set-up 


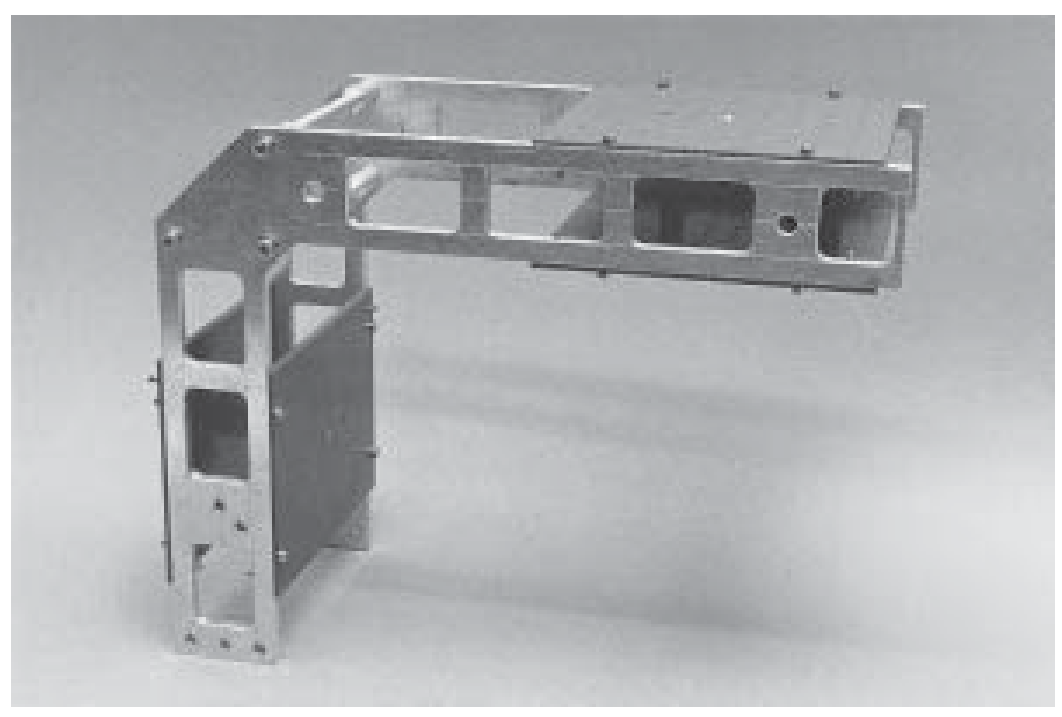

Fig. 6 The 'gamma' X-ray photogrammetry calibration frame
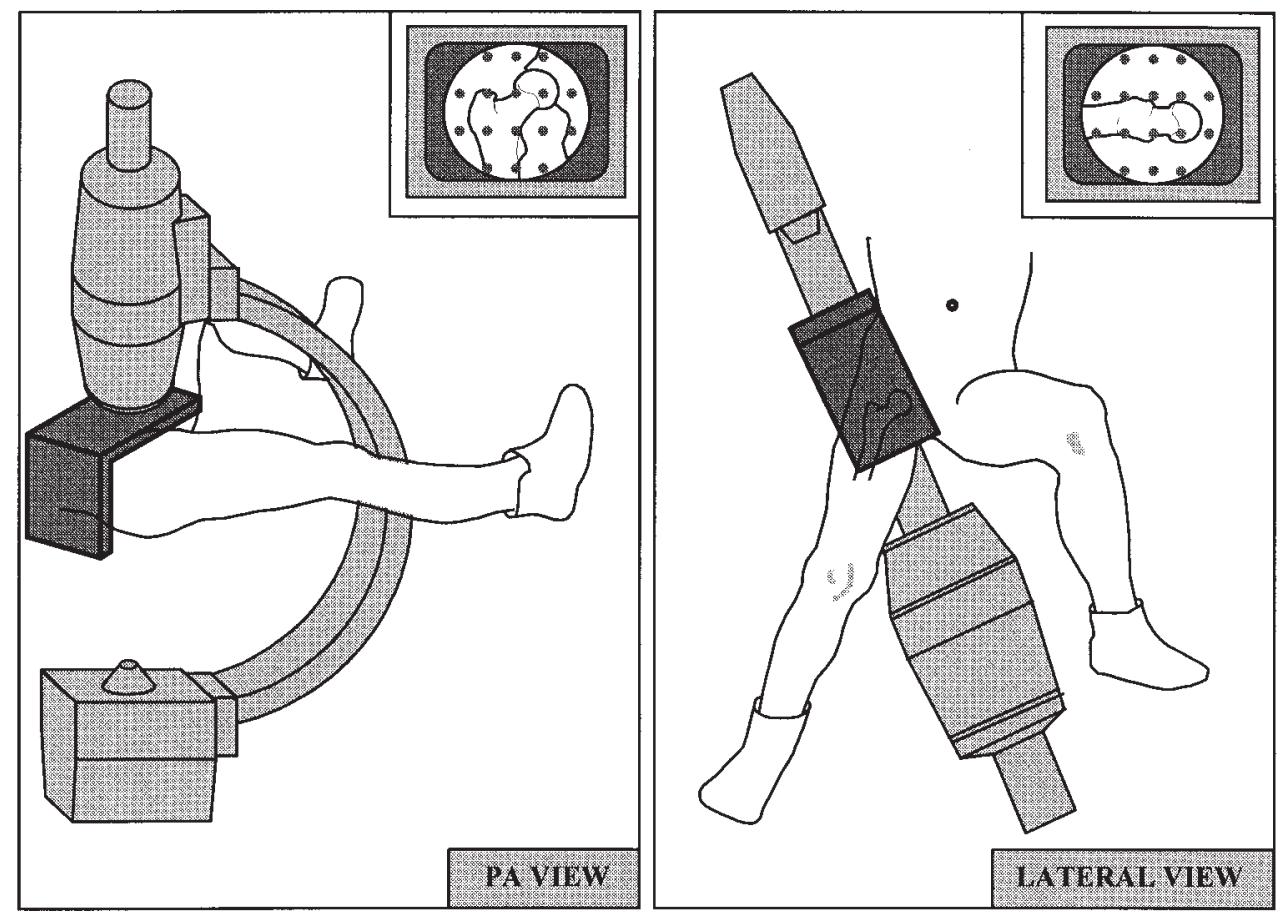

Fig. $7 \Gamma$-frame positioning in relation to patient anatomy

times, better robot compatability and compliance with sterility criteria).

\subsection{Semi-automatic image analysis}

In order to perform the photogrammetric reconstruction process, two sets of accurate input data are required: the real-world (millimetre) coordinates of the fiducial markers embedded in the radiolucent plates of the calibration frame, and their corresponding (pixel) coordinates in the intraoperative images. Given the time constraints associated with an intraoperative application, extrac- tion of the image coordinate data must be performed with the minimum level of user intervention. A semiautomatic digital image analysis scheme has therefore been implemented to 'scan' the four imaged calibration plates of the $\Gamma$ frame rapidly.

From the software perspective, the image analysis process can be significantly simplified through the provision of a carefully considered fiducial pattern. Previous research [16] suggests that the design (size, shape, etc.) of fiducial markers can have a significant effect upon the accuracy of the image measurement process. With a view to superimposing isolated circular markers on the intraoperative fluoroscopic images, grid patterns consisting 
of stainless steel ball-bearings have been embedded into the radiolucent plates of the $\Gamma$ frame. In addition to being invariant to spatial orientation, the resulting circular fiducials are also compact (i.e. have a low perimeter-area ratio), thus minimizing measurement errors associated with the digitizing process.

In order to establish corresponding real world-image point pairs, the image analysis software must be able to uniquely identify or 'label' each of the fiducial markers appearing within the field of view. Because the position of the $\Gamma$ frame is not fixed with respect to the $\mathrm{C}$-arm unit, this unique fiducial identification process can only be achieved by introducing reference or datum markers into the field of view (e.g. larger diameter ball-bearings, or washers). These reference markers also allow both the in-plane angular orientation of the $\Gamma$ frame (i.e. a grid rotation angle) and the dimensions of the grid pattern (in pixels) to be estimated. When the $\Gamma$ frame and the patient are imaged together, a typical registration image, incorporating fiducial patterns provided by an adjacent pair of calibration plates, therefore takes the form shown in Fig. 8.

The image scanning routine works by initially locating the reference markers of a particular calibration plate. The coordinate ( pixel) data provided by these reference markers then allows the 'expected' locations of the other fiducials in the relevant grid pattern to be estimated. A circular area of interest centred at each of these predicted locations is then automatically scanned, by applying an appropriate sequence of image enhancement, segmentation and pattern recognition-subpixel measurement routines. The resulting image coordinate data are then written to file in an array format.

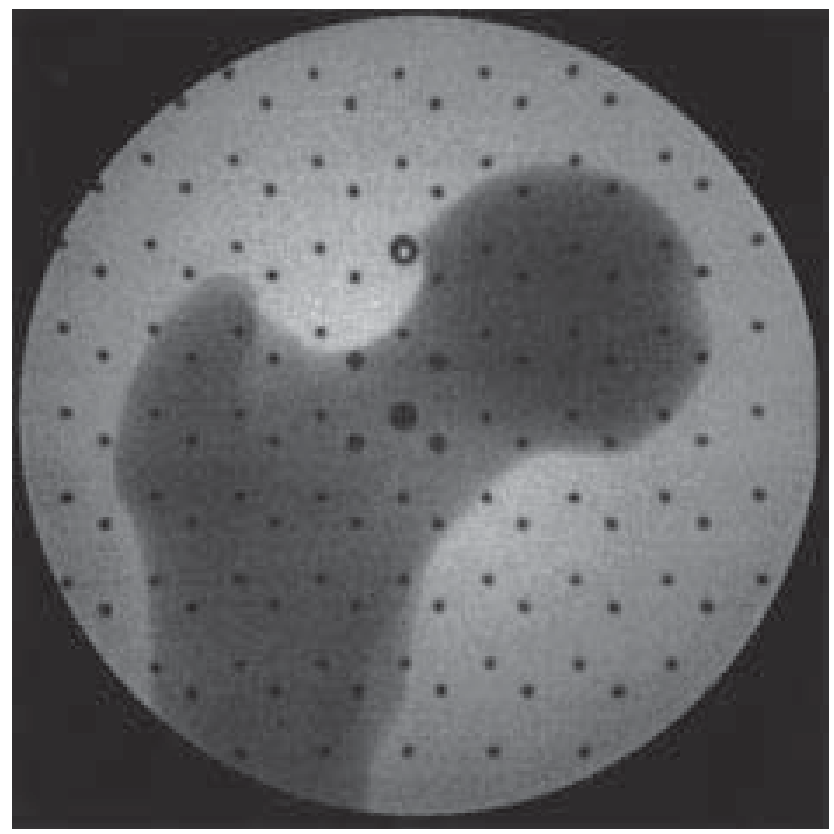

Fig. 8 A simulated $\Gamma$-frame registration image (simulated PA view of plastic femur- $\Gamma$-frame combination)

\subsection{Trajectory planning}

If it is assumed that X-rays travel in straight lines and are not deflected when passing through an object, and that the X-ray source can be approximated to a perfect point source, radiography can be considered to be a special case of central projection, in which all rays (or 'lines of sight') pass through the focus of the X-ray source (i.e. a perspective centre). When this imaging model is applied to the geometry of the $\Gamma$ frame, the photogrammetric reconstruction process takes the form shown in Fig. 9. The three-dimensional location of an imaged target point $\mathrm{T}$ is therefore obtained, with respect to the coordinate system of the $\Gamma$ frame, by reconstructing two 'intersecting' lines of sight. In practice, measurement errors ensure that the two lines of sight do not accurately intersect, and the location of the target point is estimated by calculating the point of nearest approach between the two lines of sight.

Applying vector analysis, the PA view line of sight can be determined from the location of the relevant X-ray focal point (labelled PA Focal in Fig. 9) and the point at which the desired line of sight passes through either of the PA calibration plates on the $\Gamma$ frame (i.e. PA/X or $\mathrm{PA} / \mathrm{S})$ :

$$
\boldsymbol{R}_{\mathrm{PA}}=\left(\begin{array}{c}
X_{\mathrm{PA} / \mathrm{X}} \\
Y_{\mathrm{PA} / \mathrm{X}} \\
Z_{\mathrm{PA} / \mathrm{X}}
\end{array}\right)+\lambda_{\mathrm{PA}}\left(\begin{array}{c}
X_{\mathrm{PA} \text { Focal }}-X_{\mathrm{PA} / \mathrm{X}} \\
Y_{\mathrm{PA} \text { Focal }}-Y_{\mathrm{PA} / \mathrm{X}} \\
Z_{\mathrm{PA} \text { Focal }}-Z_{\mathrm{PA} / \mathrm{X}}
\end{array}\right)
$$

where $\left(X_{\mathrm{PA} \text { Focal }}, Y_{\mathrm{PA} \text { Focal }}, Z_{\mathrm{PA} \text { Focal }}\right)$ are the coordinates of the PA view $X$-ray focal point with respect to the coordinate system of the $\Gamma$ frame, $\left(X_{\mathrm{PA} / \mathrm{X}}, Y_{\mathrm{PA} / \mathrm{X}}, Z_{\mathrm{PA} / \mathrm{X}}\right)$ are the coordinates, with respect to the coordinate system of the $\Gamma$ frame, of the point at which the desired line of sight passes through the PA view XRII calibration plane (defined by the calibration plate nearest to the $\mathrm{X}$-ray image intensifier) and $\lambda_{\mathrm{PA}}$ is a constant.

Similarly, the lateral-to-medial view line of sight can be calculated from the coordinates of the lateral view X-ray focal point (labelled Lat. Focal in Fig. 9) and either of the intersection points with the lateral-to-medial view calibration plates of the $\Gamma$ frame (i.e. Lat/X or Lat/S):

$$
\boldsymbol{R}_{\text {Lat }}=\left(\begin{array}{c}
X_{\text {Lat } / \mathrm{X}} \\
Y_{\text {Lat } / \mathrm{X}} \\
Z_{\text {Lat } / \mathrm{X}}
\end{array}\right)+\lambda_{\text {Lat }}\left(\begin{array}{c}
X_{\text {Lat Focal }}-X_{\text {Lat } / \mathrm{X}} \\
Y_{\text {Lat Focal }}-Y_{\text {Lat } / \mathrm{X}} \\
Z_{\text {Lat Focal }}-Z_{\text {Lat } / \mathrm{X}}
\end{array}\right)
$$

where $\left(X_{\text {Lat Focal }}, Y_{\text {Lat Focal }}, Z_{\text {Lat Focal }}\right)$ are the coordinates of the lateral-to-medial view $X$-ray focal point with respect to the coordinate system of the $\Gamma$ frame, $\left(X_{\mathrm{Lat} / \mathrm{X}}\right.$, $\left.Y_{\text {Lat } / \mathrm{X}}, Z_{\text {Lat } / \mathrm{X}}\right)$ are the coordinates, with respect to the coordinate system of the $\Gamma$ frame, of the point at which the desired line of sight passes through the lateral-to-medial view XRII calibration plane and $\lambda_{\text {Lat }}$ is a constant.

As a consequence, the photogrammetric reconstruction process requires the (pixel) coordinates of the target point to be measured in both images. In other words, 


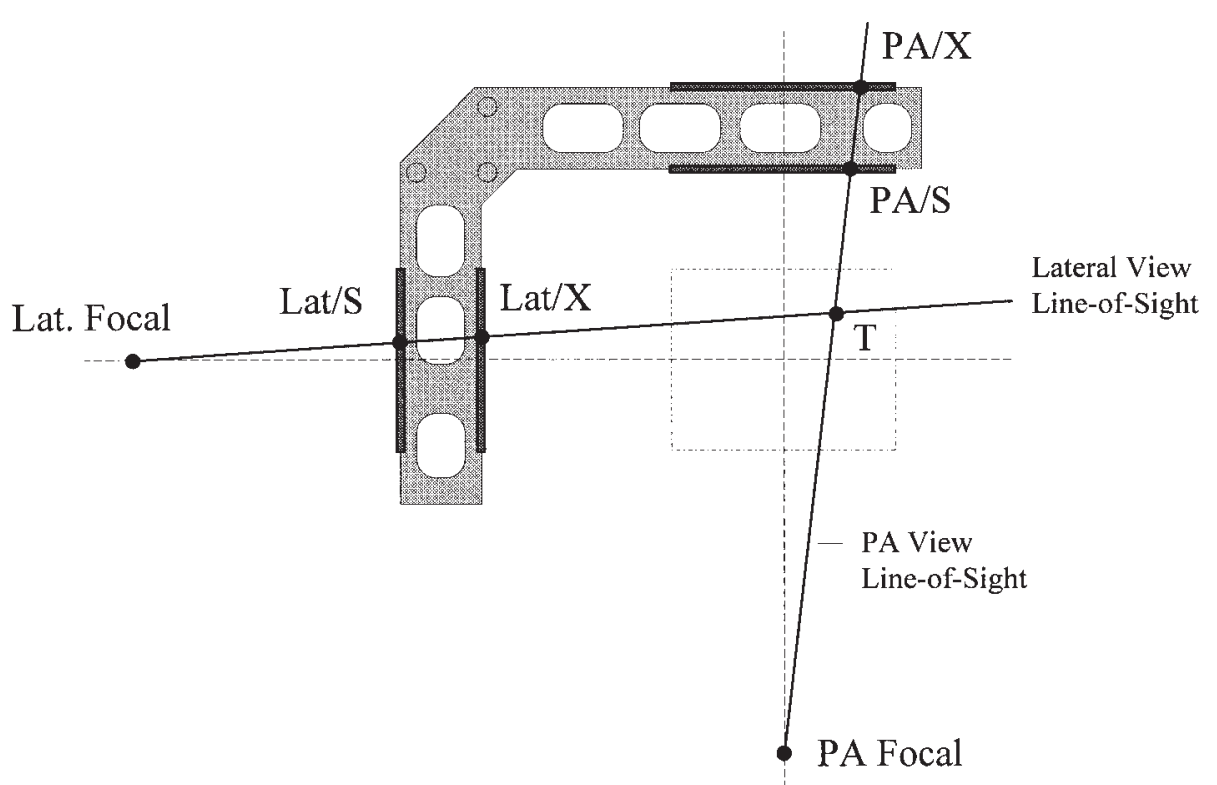

Fig. 9 The $\Gamma$-frame-based photogrammetric reconstruction process

having initially located the target point in the PA view, the corresponding point must then be identified in the lateral-to-medial view. Since the PA and lateral-tomedial views are orthogonal to one another and as such are very dissimilar, this complicated image-matching problem is potentially a major source of errors. With a view to improving the accuracy of the guide wire insertion process, the computer-assisted trajectory planning scheme summarized by Fig. 10 has therefore been developed.

Owing to the simplicity of the desired guide wire trajectory, an interactive indication process, which

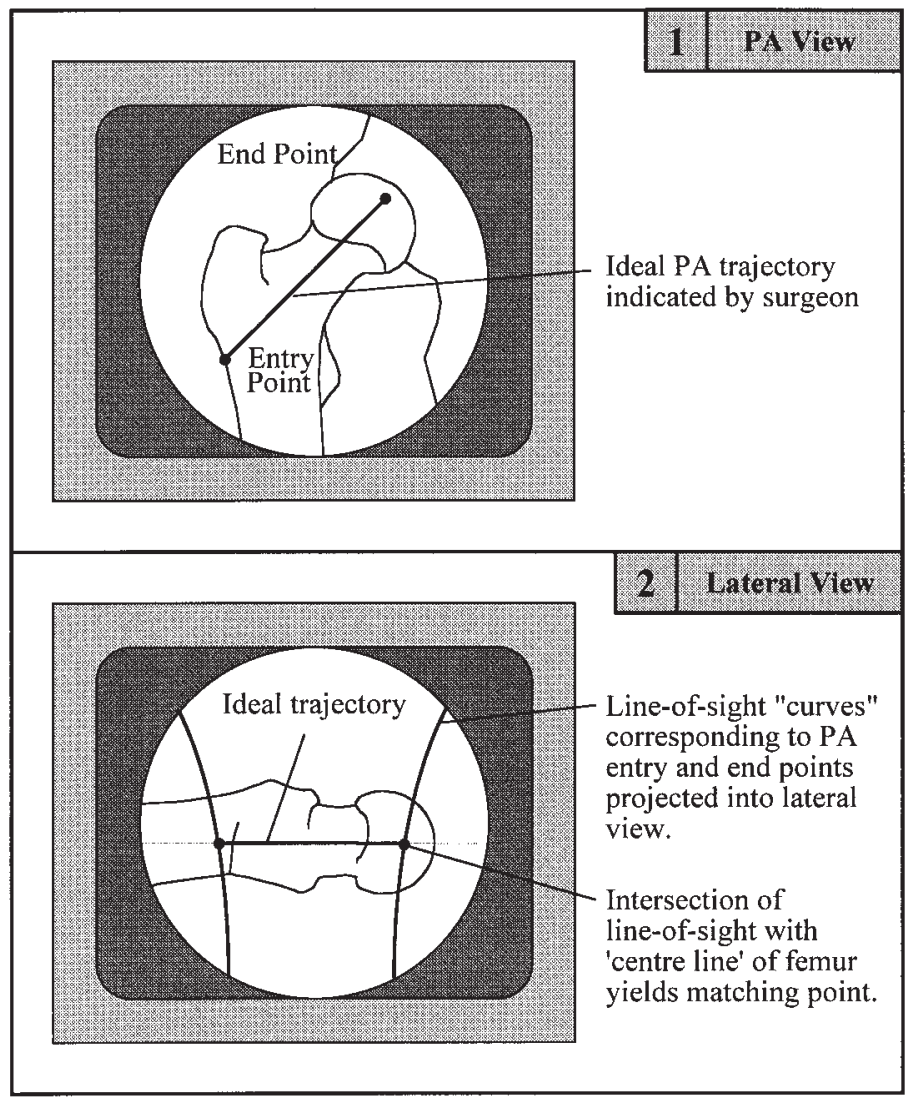

Fig. 10 Computer-assisted planning of the guide wire trajectory 
requires the selection of an entry point and an end point in both views, has been adopted. The surgeon is therefore provided with a PC-mouse-driven human-computer interface, which allows trajectories to be indicated as graphical overlays on top of the calibrated fluoroscopic images. Accordingly, the ideal entry and end points are initially indicated on the PA view. The trajectory planning software then uses the pixel coordinates of these entry and end points, and the calibration data provided by the $\Gamma$ frame, to reconstruct the corresponding lines of sight. The appropriate world-image transformations are then used to project the two lines of sight into the lateral-to-medial image. As indicated by Fig. 10, the surgeon then uses these projected lines to guide selection of the entry and end points in the lateral-to-medial view.

The main prerequisite of this trajectory planning scheme is knowledge of the PA and lateral-to-medial view X-ray source locations. Given that the central projection model assumes that all lines of sight pass through the X-ray focal point, as illustrated in Fig. 11, effective focal point locations $\mathrm{F}$ can be obtained from the intersection of several reconstructed lines of sight. Having scanned the appropriate registration image, the realworld and image coordinates of the fiducials provided by the XRII calibration plate (i.e. the calibration plate nearest to the X-ray image intensifier) are known. Lines of sight which pass through these XRII plate fiducials and the desired effective focal point can therefore be reconstructed by calculating the points at which they intersect the adjacent source calibration plate (i.e. the calibration plate nearest to the X-ray source).

In practice, these source plate intersection points are obtained by applying the distortion compensation (image-world transformation) scheme described in Section 4.4 to the image coordinates of the XRII fiducials and the calibration data provided by the source plate fiducials. Having reconstructed several (typically 16-25) lines of sight, the required location of the effective focal point is estimated by performing a least- squares calculation, which attempts to minimize the sum of the distances from each of the lines of sight to a given point.

Knowledge of the PA view effective focal point allows the lines of sight which pass through the entry and end points indicated by the surgeon in the PA image to be reconstructed. However, when projecting these lines of sight into the lateral-to-medial image, the effects of image distortion must be taken into account. As illustrated in Fig. 12, each of the PA view lines of sight is therefore sampled at fixed increments. Lines of sight which pass through the lateral view effective focal point and each of these sample points are then reconstructed, thus allowing the points at which these new lines of sight intersect the lateral view source calibration plate of the $\Gamma$ frame to be calculated. These real-world intersection points are then converted into pixel coordinates by applying localized world-image transformations. The desired 'image-matching lines' are then obtained by drawing line segments between the resulting image (pixel) locations. In practice, due to the effects of image distortion, connecting the projected points in this manner actually causes a pair of matching 'curves' to be overlaid into the lateral-to-medial image.

\subsection{Compensation for image distortion effects}

The mobile C-arm fluoroscopy unit allows direct visualization of dynamic X-ray images, via the use of an $\mathrm{X}$-ray image intensifier tube. X-rays entering the input window of this device are initially absorbed by a fluorescent input phosphor, resulting in the production of light quanta (i.e. photons). These photons then hit a photocathode, which is in contact with the input phosphor, where they cause photoelectrons to be emitted. The photoelectrons are then accelerated by an electrostatic field and are focused by an electron-optical system towards an anode aperture. After passing through this 'electron focus', the photoelectrons then strike an output

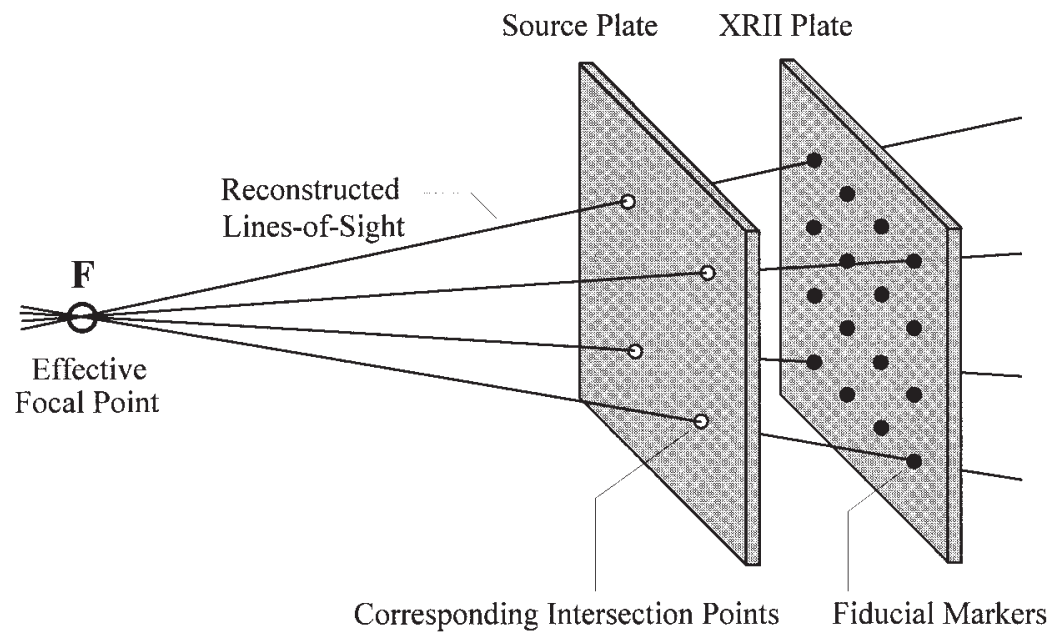

Fig. 11 Estimation of the effective X-ray focal point 


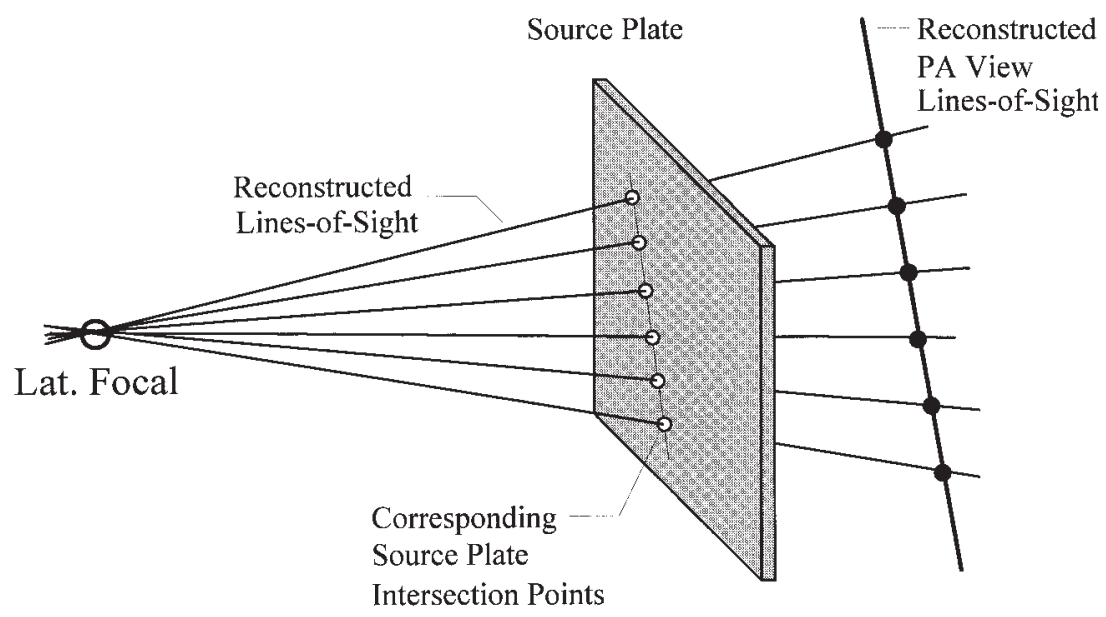

Fig. 12 Projecting a PA line of sight into the lateral view

phosphor where they produce a visible-light image. The required video images are then obtained from the output window of the X-ray image intensifier tube using either a Vidicon camera (in the case of older C-arm units) or a charge-coupled device camera (in the case of modern $\mathrm{C}$-arm units). The amplified output from the camera is then transmitted as a video signal to a conventional television monitor, thus allowing real-time viewing of the fluroscopic images. Alternatively, as in the case of the robotic application described in this paper, a videolink can be established with this display monitor, thus allowing digital sampling of the video signal.

Modern image intensifier tubes employ an electrostatic lens design, in which the photocathode and anode are both curved spherically and have a common centre point. As a result, the electron 'image' formed at the photocathode is produced by central projection from the $\mathrm{X}$-ray source on to a curved surface. The outcome of this image formation process is a fluoroscopic image which exhibits noticeable spatial distortion and associated intensity variations. This phenomenon is generally referred to as 'pincushion distortion'.

Since the magnitude of the pincushion distortion effect is usually too significant to be neglected, fluoroscopybased X-ray photogrammetry requires an additional image distortion correction process to be performed. If it is assumed that image distortion is symmetrical over the entire field of view, a global polynomial function can be used to estimate the relationship between output (distorted image) and input (real-world) coordinates [17-19]. The coefficients of such a relationship are generally determined by imaging a calibration grid which provides a series of fiducial points with known (real-world) coordinates. However, in reality, image distortion is often found to be asymmetrical across the field of view. A number of workers have therefore proposed the use of correction schemes employing a mosaic of localized correction functions [20-22]. Several analytical techniques, based upon mathematical modelling of the projection process, have also been reported [23-25].

H00199 (C) IMechE 2000
Unfortunately, it can easily be demonstrated that photocathode curvature is not the only source of image distortion. Figure 13 shows a 'difference' or 'subtraction' image which was produced by digitally subtracting a ' $\mathrm{PA}$ ' $\left(\mathrm{C}\right.$-arm arc rotation of $0^{\circ}$ ) image of a calibration object from the corresponding 'lateral' (C-arm arc rotation of $90^{\circ}$ ) image of the object. Given that the calibration object, which consists of an aluminium plate into which a grid of holes has been accurately drilled, was rigidly attached to the image intensifier housing, these PA and lateral images should be identical, thus producing a blank 'difference' image. However, this is evidently not the case, with the 'distorted' locations of both the PA (white) and the lateral (black) fiducials being clearly visible in Fig. 13.

These frequently overlooked discrepancies are mainly

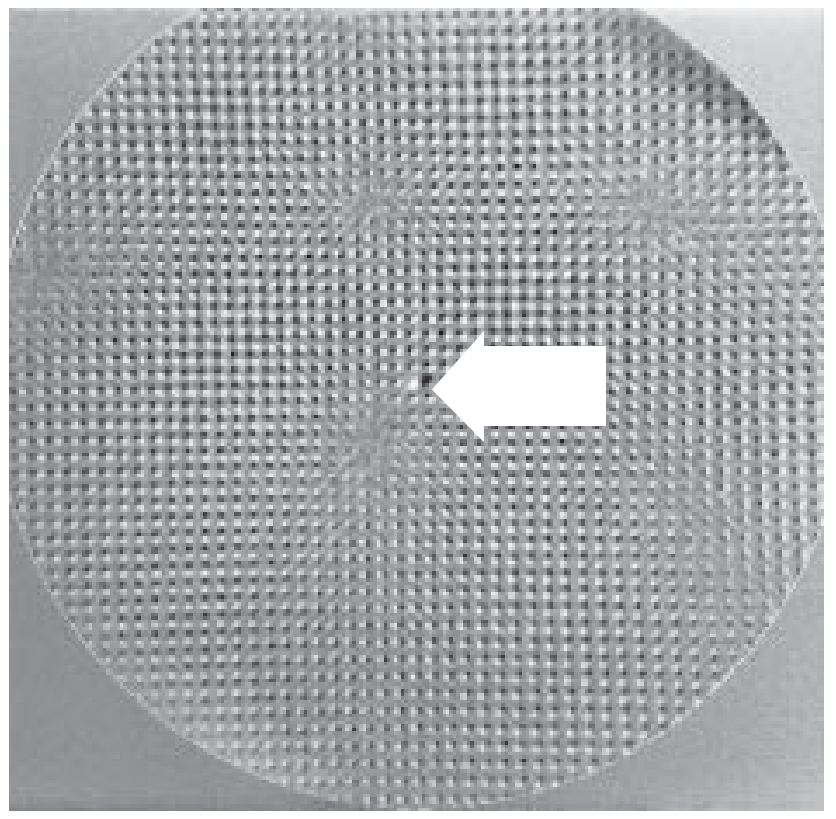

Fig. 13 A typical PA-lateral subtraction image of the calibration object. The white arrow indicates the location of the centre marker of the calibration object 
attributable to the influence of the geomagnetic field upon the electrons inside the image intensifier tube. When an electron travels through a magnetic field, it experiences an induced force which, in accordance with the Lorentz force law [26], acts at right angles to both its velocity and the magnetic field. In the case of the image intensifier tube, this induced force deflects electrons from their true trajectories and therefore represents an additional source of image distortion. The geometrical form of this image distortion depends upon the orientation of the image intensifier tube with respect to the Earth's magnetic field [27].

If the image intensifier tube is perpendicular to the geomagnetic field, the induced force causes shifting (displacement) of the image in one direction; a difference of 2-3 mm ( 8 pixels) exists between the location of the central grid hole (indicated by the white arrow in Fig. 13) in the PA and lateral views used to create the subtraction image. By contrast, for the parallel orientation case, the induced force causes rotation of the image. A characteristic S-shaped warping is therefore observed because this image rotation effect is partially compensated for by the 'magnetic' shield which is placed around the sides of most image intensifier tubes.

In addition to the geomagnetic distortion effect, the possibility of additional magnetic influences in the operating room cannot be overlooked. The image intensifier housing of the $\mathrm{C}$-arm unit is by necessity frequently placed in close proximity to large metallic objects (e.g. the operating table). Electromagnetic interference caused by operating room equipment is also widely acknowledged as being a significant problem. In relation to the mobile C-arm fluoroscopy unit, the magnetic-related image distortion effects are therefore highly unpredictable and, as such, cannot be modelled off line (i.e. preoperatively).

Since the photogrammetric reconstruction process is achieved via the use of the $\Gamma$ frame, the same frame has also been used to provide calibration data for the distortion compensation process. In order to provide compensation for image distortion effects, the calculation of the appropriate calibration plate intersection points is therefore performed using the localized interpolation scheme summarized by Fig. 14 .

Once the image analysis task has been performed, both the image (pixel) and the real-world (millimetre) coordinates of the fiducial markers appearing within the field of view are known. By comparing the measured image coordinates of the target point $\mathrm{T}$ with the image coordinates of the fiducial markers of the relevant calibration plate, it is therefore possible to determine the three closest fiducial markers (fiducials 5, 6 and 8 in Fig. 14), thereby defining a bounding triangle. A localized image-world transformation can then be defined for this specific triangular subregion of the image:

$$
[\mathbf{W}]=[\mathbf{A}][\mathbf{P}]
$$

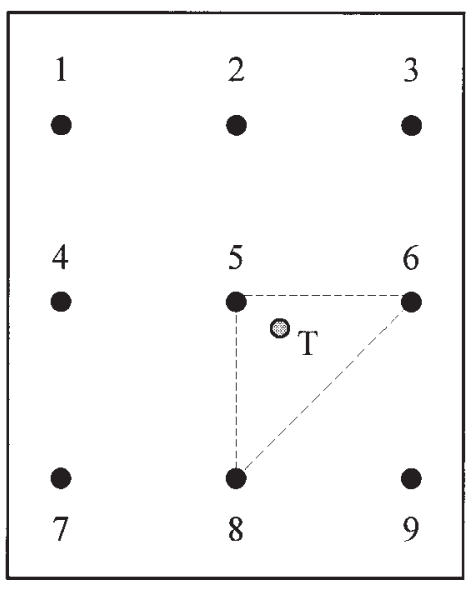

Fig. 14 Division of calibration image into triangular subregions. Numbered discs represent imaged fiducial markers

where matrix [W] contains the real-world coordinates $\left(X_{i}, Y_{i}, Z_{i}\right)$ of the relevant fiducials, matrix $[\mathbf{P}]$ contains the image (pixel) coordinates $\left(x_{i}, y_{i}\right)$ of the relevant fiducials and matrix $[\mathbf{A}]$ contains the required linear interpolation function coefficients, obtained by performing the calculation

$$
[\mathbf{A}]=[\mathbf{W}][\mathbf{P}]^{-1}
$$

For the scenario shown in Fig. 14, the image-world transformation would therefore be defined by

$$
\left[\begin{array}{ccc}
X_{5} & X_{6} & X_{8} \\
Y_{5} & Y_{6} & Y_{8} \\
Z_{5} & Z_{6} & Z_{8}
\end{array}\right]=\left[\begin{array}{lll}
A_{1,1} & A_{1,2} & A_{1,3} \\
A_{2,1} & A_{2,2} & A_{2,3} \\
A_{3,1} & A_{3,2} & A_{3,3}
\end{array}\right]\left[\begin{array}{ccc}
x_{5} & x_{6} & x_{8} \\
y_{5} & y_{6} & y_{8} \\
1 & 1 & 1
\end{array}\right]
$$

Having determined the appropriate transformation coefficients $\left[A_{i, j}\right]$, the intersection of the line of sight of the target point with the calibration plate can then be obtained from

$$
\left[\begin{array}{c}
X_{\text {target }} \\
Y_{\text {target }} \\
Z_{\text {target }}
\end{array}\right]=\left[\begin{array}{lll}
A_{1,1} & A_{1,2} & A_{1,3} \\
A_{2,1} & A_{2,2} & A_{2,3} \\
A_{3,1} & A_{3,2} & A_{3,3}
\end{array}\right]\left[\begin{array}{c}
x_{\text {target }} \\
y_{\text {target }} \\
1
\end{array}\right]
$$

where $\left(x_{\text {target }}, y_{\text {target }}\right)$ are the measured (distorted) pixel coordinates of the target point and $\left(X_{\text {target }}, Y_{\text {target }}\right.$, $Z_{\text {target }}$ ) are the ('undistorted') coordinates of the desired intersection point.

The main advantage offered by this $\Gamma$-frame-based technique is that data from the actual intraoperative images are used to perform the distortion compensation process. Consequently, assumptions do not have to be made with regards to the magnetic influences in the operating room, the internal geometry of the image intensifier tube or the orientation of the C-arm unit. Each individual fluoroscopic image is therefore cali- 
brated using data that are valid for the specific imaging geometry used at the time of its acquisition.

\section{DISCUSSION}

Osteosynthesis procedures involve interactions with inherently rigid structures (i.e. bones), and are therefore ideal candidates for computer and robotic assistance. Predictably, research groups working in this application area have initially targeted the most frequently performed and the most technically demanding internal fixation procedures. As a consequence, in addition to studies of the hip fracture repair procedure $[9,28,29]$, a variety of spinal fusion $[\mathbf{3 0}, \mathbf{3 1}]$ and femoral shaft fracture repair $[\mathbf{1 0}, \mathbf{3 2}, 33]$ procedures are also currently under investigation. Given that each of these osteosynthesis studies involves a fluoroscopically guided drilling process, the problem of incorporating fluoroscopic images into an intraoperative registration strategy has had to be addressed.

In the case of the studies targeted at the distal interlocking stage of the intramedullary nailing of femoral shaft fractures [32, 33], an optoelectronic position sensor (Optotrak $\left.^{(i 0}\right)$ has been used to establish a common or 'operating room' coordinate system. However, trials have shown that maintaining an unobstructed line of sight between the Optotrak sensor and the infrared lightemitting diode array mounted on the $\mathrm{C}$-arm unit may not always be practicable in the cluttered operating room environment [32].

Lavallée et al.[31] have investigated several intraoperative registration strategies with a view to assisting the insertion of pedicle screws during spinal fusion procedures. Under their X-ray imaging-based registration scenario [34], a robotic arm is used to position a radiolucent calibration plate within the field of view of a fluoroscopy unit. Having acquired an image of the calibration plate, the process is then repeated by moving the plate to a second location within the field of view. However, in order to accommodate this technique, which allows the patient and the calibration object to be imaged separately, two intraoperative fluoroscopy systems must be used.

The registration strategy described in this paper represents an attempt to adopt a proven radiography-based metrology technique to meet the requirements of an intraoperative (fluoroscopy-based) application. In order to achieve compatibility with both patient anatomy and the mobile C-arm fluoroscopy unit, an extrapolationbased photogrammetric reconstruction process has been advocated. Tests involving a prototype $\Gamma$ frame indicate that the loss of precision associated with this deviation from the 'classical' photogrammetry approach is not significant in relation to the accuracy requirements of the guide wire insertion process.

A true measure of the performance of an X-ray photo- grammetry system is gained by reconstructing the locations of fiducials which are not used during the calibration-registration process. In order to provide these 'independent' test points a Perspex calibration object, into which four ball-bearings are embedded, has been manufactured. Attaching this calibration object to the prototype $\Gamma$ frame therefore introduces four test points into the field of view. The locations of these test points, with respect to the coordinate system of the $\Gamma$ frame, have been independently verified using theodolites. As such, an indication of the errors associated with the photogrammetric reconstruction process can be obtained by interactively indicating the four test points in both a 'PA' image and a 'lateral-to-medial' image.

As indicated in Table 1 (second row of results), when photogrammetric reconstruction is performed using the localized distortion compensation technique described in Section 4.4, tests involving the prototype $\Gamma$ frame give a maximum (absolute) reconstruction error of the order of $0.88 \mathrm{~mm}$. Applying the same reconstruction software to a 'classical' biplane X-ray photogrammetry calibration frame, which defines an identical measurement volume to that of the prototype $\Gamma$ frame, has led to maximum reconstruction errors of the order of $0.48 \mathrm{~mm}$. The switch from interpolation- to extrapolation-based reconstruction therefore accounts for an 'acceptable' loss of precision of approximately $0.4 \mathrm{~mm}$.

With a view to evaluating the effectiveness of the image distortion compensation scheme, tests have also been performed without adequate distortion compensation. These tests involved the use of reconstruction software based upon global distortion compensation functions, which were derived by applying a leastsquares fitting to the data provided by all the fiducials appearing within the field of view. Given the asymmetrical (localized) nature of the image distortion effects, this approach can lead to significant over-compensation or under-compensation. The resulting loss of precision, as indicated by the third row of results in Table 1 , therefore provides a strong reminder of the potential pitfalls when applying an extrapolation-based reconstruction technique to a non-linear imaging system.

A more significant validation of the proposed registration technique has been obtained by performing simulated guide wire insertion procedures on phantom (plastic) femurs containing pre-drilled holes. During these system integration trials, the improved $\Gamma$-frame design shown in Fig. 6 was used to derive drilling trajectories, which were then downloaded to the system's robot controller. The prototype robotic manipulator then aligned the guide wire with the reconstructed drilling trajectory, prior to feeding it into the pre-drilled hole in the plastic femur.

The results of the first six guide wire insertion trials are summarized in Fig. 15. From this figure it can be seen that every attempt to insert the guide wire of $2.5 \mathrm{~mm}$ diameter into the pre-drilled hole of $8 \mathrm{~mm}$ diameter in 
Table 1 Typical photogrammetric reconstruction data, where the absolute error values $E=\sqrt{\partial x^{2}+\partial y^{2}+\partial z^{2}}$

\begin{tabular}{lllllll}
\hline $\begin{array}{l}\text { Calibration } \\
\text { frame geometry }\end{array}$ & $\begin{array}{l}\text { Image distortion } \\
\text { compensation }\end{array}$ & $\begin{array}{l}\text { Test point } 1 \\
(\mathrm{~mm})\end{array}$ & $\begin{array}{l}\text { Test point 2 } \\
(\mathrm{mm})\end{array}$ & $\begin{array}{l}\text { Test point 3 } \\
(\mathrm{mm})\end{array}$ & $\begin{array}{l}\text { Test point 4 } \\
(\mathrm{mm})\end{array}$ & $\begin{array}{l}\text { Maximum error } \\
(\mathrm{mm})\end{array}$ \\
\hline Biplane & Localized & 0.48 & 0.30 & 0.33 & 0.30 & 0.48 \\
$\Gamma$ frame & Localized & 0.78 & 0.42 & 0.88 & 0.84 & 0.88 \\
$\Gamma$ frame & Global & 2.98 & 2.60 & 2.99 & 3.40 & 3.40 \\
\hline
\end{tabular}

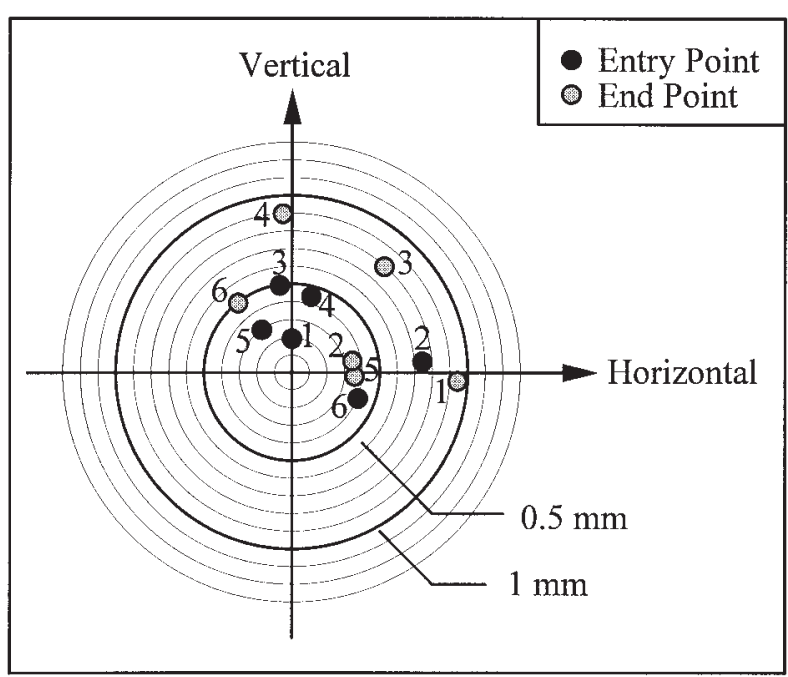

Fig. 15 Location of the guide wire tip in relation to the desired entry and end points

the plastic bone was successful. In each case, the tip of the guide wire was within $1 \mathrm{~mm}$ of the desired entry point (i.e. the centre of the pre-drilled hole) and had an angular deviation with respect to the axis of the predrilled hole of less than $0.6^{\circ}$. Having subsequently demonstrated this level of precision during a large number of follow-up trials, a more realistic series of tests involving flesh-covered porcine femurs is planned for the near future.

Thus, in summary, the $\Gamma$-frame-based technique described in this paper provides an accurate low-cost non-invasive registration option, which fully adheres to 'operating room compatibility' criteria. By extending the technique to include quantification of the trajectory planning process, it has also been possible to overcome the existing depth perception problem. Significantly, given that the $\Gamma$ frame supports the standard biplane fluoroscopic examination process, the technique is also applicable to a wide range of osteosynthesis procedures; the preliminary results from a parallel study tend to suggest that the $\Gamma$ frame is equally applicable to roboticassisted distal interlocking of femoral shaft fractures.

\section{ACKNOWLEDGEMENTS}

This research project has been partially funded by the Wishbone Trust of the British Orthopaedic Association. The authors would also like to take this opportunity to thank Professor J. R. Hewit (Department of Applied Physics and Electronics and Mechanical Engineering, University of Dundee), Mr R. G. Shedden (Consultant Orthopaedic Surgeon, Mayday University Hospital, Croydon Area Health Authority) and $\mathrm{Mr}$ M. J. Hochrainer (MSc student) for their contribution to the project.

\section{REFERENCES}

1 Klenerman, L. The young patient with a fractured neck of femur. Br. Med. J., 1985, 290(6486), 1928.

2 Riggs, B. L. and Melton, L. J. Involutional osteoporosis. New Engl. J. Medicine, 1986, 314(26), 1676-1686.

3 Ganz, R. Proximal femur. In Manual of Internal Fixation: Techniques Recommended by the AO-ASIF Group (Eds M. E. Muller, M. Allgower, R. Schneider and H. Willenegger), 3rd edition, 1991, pp. 519-534 (SpringerVerlag, Berlin).

4 Davis, T. R. C., Sher, J. L., Horsman, A., Simpson, M., Porter, B. B. and Checketts, R. G. Intertrochanteric femoral fractures: mechanical failure after internal fixation. $J$. Bone Jt Surgery (Br.), 1990, 72(1), 26-31.

5 Mainds, C. C. and Newman, R. J. Implant failures in patients with proximal fractures of the femur treated with a sliding screw device. Injury, 1989, 20(2), 98-100.

6 Thomas, A. P. Dynamic hip screws that fail. Injury, 1991, $22(1), 45-46$.

7 Gundle, R., Gargan, M. F. and Simpson, A. H. R. W. How to minimise failures of fixation of unstable intertrochanteric fractures. Injury, 1995, 26(9), 611-614.

8 Parker, M. J. Cutting-out of the dynamic hip screw related to its position. J. Bone Jt Surgery, 1992, 74(4), 625.

9 Bouazza-Marouf, K., Browbank, I. and Hewit, J. R. Robotic-assisted internal fixation of femoral fractures. Proc. Instn Mech. Engrs, Part H, Journal of Engineering in Medicine, 1995, 209(H1), 51-58.

10 Bouazza-Marouf, K., Browbank, I. and Hewit, J. R. Robotassisted invasive orthopaedic surgery. Mechatronics, June 1996, 6(4), 381-397.

11 Laros, G. S. Intertrochanteric fractures. In Surgery of the Musculoskeletal System, Vol. 3 (Ed. C. M. Evarts), 1990, pp. 2613-2639 (Churchill Livingstone, New York).

12 Ong, F. R. and Bouazza-Marouf, K. Drilling of bone: a robust automatic method for the detection of drill bit break-through. Proc. Instn Mech. Engrs, Part H, Journal of Engineering in Medicine, 1998, 212(H3), 209-221.

13 Ryd, L. Roentgen stereophotogrammetric analysis of prosthetic fixation in the hip and knee joint. Clin. Orthop. Related Res., 1992, 276, 56-65.

14 Turner-Smith, A. R., White, S. P. and Bulstrode, C. X-ray 
photogrammetry of artificial hip joints. In Close-Range Photogrammetry Meets Machine Vision, Proceedings of SPIE-The International Society for Optical Engineering, Zurich, Switzerland, 3-7 September 1990, Vol. 1395(1), 1990, pp. 587-594 (SPIE, Bellingham, Washington).

15 Hunter, J. C., Baumrind, S., Genant, H. K., Murray, W. R. and Ross, S. E. The detection of loosening in total hip arthroplasty: description of a stereophotogrammetric computer assisted method. Investigative Radiology, 1979, 14(4), 323-329.

16 Bose, C. B. and Amir, I. Design of fiducials for accurate registration using machine vision. IEEE Trans. Pattern Analysis Mach. Intell., 1990, 12(12), 1196-1200.

17 Casperson, L. W., Spiegler, P. and Grollman, J. H. Characterisation of aberrations in image-intensified fluoroscopy. Med. Physics, 1976, 3(2), 103-106.

18 Guggenheim, N., Doriot, P. A., Dorsaz, P. A., Descouts, P. and Rutishauser, W. Spatial reconstruction of coronaryarteries from angiographic images. Physics Medicine Biology, 1991, 36(1), 99-110.

19 Haaker, P., Klotz, E., Koppe, R. and Linde, R. Real-time distortion correction of digital X-ray II/TV systems: an application example. In Proceedings of the Image Processing II Meeting, Hamburg, Germany, 19-21 September 1988, Proceedings of SPIE-The International Society for Optical Engineering, Vol. 1027, 1989, pp. 261-266 (SPIE, Bellingham, Washington).

20 Butler, D. A. and Pierson, P. K. A distortion-correction scheme for industrial machine-vision applications. IEEE Trans. Robotics Automation, August 1991, 7(4), 546-551.

21 Boone, J. M., Seibert, J. A., Barrett, W. A. and Blood, E. A. Analysis and correction of imperfections in the image intensifier-TV-digitizer imaging chain. Med. Physics, 1991, 18(2), 236-242.

22 Reimann, D. A. and Flynn, M. J. Automatic distortion correction of X-ray image intensifier images. In Conference Record of the IEEE Nuclear Science Symposium and Medical Imaging Conference, Orlando, Florida, 25-31 October 1992, pp. 1339-1341 (IEEE, New York).

23 Rudin, S., Bednarek, D. R. and Wong, R. Accurate characterisation of image-intensifier distortion. Med. Physics, 1991, 18(6), 1145-1151.

24 Pietka, E. and Huang, H. K. Correction of aberration in image-intensifier systems. Computerised Med. Imaging Graphics, 1992, 16(4), 253-258.

25 Chakraborty, D. P. Image intensifier distortion correction. Med. Physics, 1987, 14(2), 249-252.

26 Duffin, W. J. Electricity and Magnetism, 1980 (McGrawHill, Maidenhead, Berkshire).
27 Solzbach, U., Wollschläger, H., Zeiher, A. and Just, H. Optical distortion due to geomagnetism in quantitative angiography. Comput. Cardiology, September 1988, 355357.

28 Mohsen, A. M. M. A., Cain, T. J., Karpinski, M. R. K., Sherman, K. P., Howell, F. R., Phillips, R., Viant, W. J., Griffiths, J. G., Dyer, K. D. F. and Wells, D. P. M. The basic orthopaedic principle and the non-invasive intelligent orthopaedic guide concept. In Proceedings of the Second International Workshop on Mechatronics in Medicine and Surgery (MediMec '95), Bristol, 6-9 September 1995, pp. $47-57$.

29 Finlay, P. A. ORTHOSISTA: an active surgical localiser for assisting orthopaedic fracture fixation. In Proceedings of the Second Annual International Symposium on Medical Robotics and Computer Assisted Surgery, Baltimore, Maryland, 4-7 November 1995, pp. 203-207.

30 Santos-Munné, J. J., Peshkin, M. A., Mirkovic, S., Stulberg, D. S. and Kienzle, T. C. A stereotactic and robotic system for pedicle screw placement. In Interactive Technology and the New Paradigm for Healthcare (Eds R. M. Satava, K. Morgan, H. B. Sieburg, R. Mattheus and J. P. Christensen), Studies in Health Technology and Informatics Vol. 18, 1995, Ch. 66, pp. 326-333 (IOS Press, Amsterdam).

31 Lavallée, S., Troccaz, J., Sautot, P., Mazier, B., Cinquin, P., Merloz, P. and Chirossel, J.-P. Computer-assisted spinal surgery using anatomy-based registration. In Computer-Integrated Surgery (Eds R. H. Taylor, S. Lavallée, G. C. Burdea and R. Mösges), 1996, pp. 425-449 (MIT Press, Cambridge, Massachusetts).

32 Viant, W. J., Phillips, R., Griffiths, J. G., Ozanian, T. O., Mohsen, A. M. M. A., Cain, T. J., Karpinski, M. R. K. and Sherman, K. P. A computer assisted orthopaedic surgical system for distal locking of intramedullary nails. Proc. Instn Mech. Engrs, Part H, Journal of Engineering in Medicine, 1997, 211(H4), 293-300.

33 Hofstetter, R. Fluoroscopy based surgical navigation for femoral fracture fixation. Second Computer-Assisted Orthopaedic Surgery Symposium, Bern, Switzerland, 7-9 November 1996.

34 Hamadeh, A., Sautot, P., Lavallée, S. and Cinquin, P. Towards automatic registration between $\mathrm{CT}$ and X-ray images: co-operation between $3 \mathrm{D} / 2 \mathrm{D}$ registration and $2 \mathrm{D}$ edge detection. In Proceedings of the Second Annual International Symposium on Medical Robotics and Computer Assisted Surgery, Baltimore, Maryland, 4-7 November 1995, pp. 39-46. 